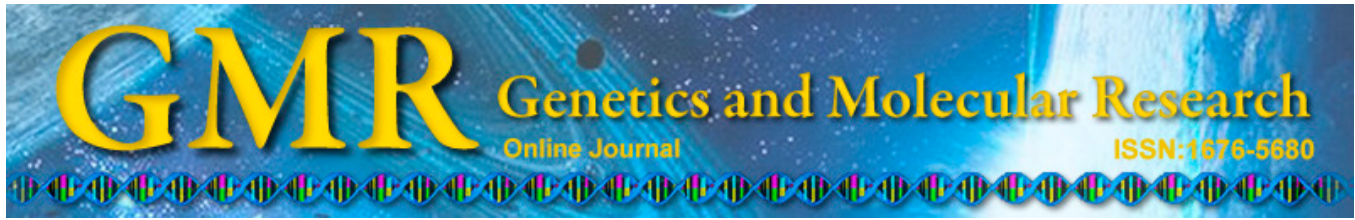

\title{
Association between the rs189037 single nucleotide polymorphism in the $A T M$ gene promoter and cognitive impairment
}

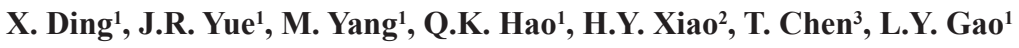 \\ and B.R. Dong ${ }^{1}$ \\ ${ }^{1}$ Department of Geriatrics, West China Hospital, Sichuan University, \\ Chengdu, Sichuan Province, China \\ ${ }^{2}$ Lab for Aging Research, Center for Medical Stem Cell Biology, \\ State Key Laboratory of Biotherapy, West China Hospital, Sichuan University, \\ Chengdu, China \\ ${ }^{3}$ Laboratory of Stem Cell Biology and Department of Geriatrics, \\ State Key Laboratory of Biotherapy, West China Hospital, \\ West China Medical School, Sichuan University, Chengdu, China \\ Corresponding authors: J.R. Yue/ B.R. Dong \\ E-mail: yuejirong11@hotmail.com / birongdong@163.com
}

Genet. Mol. Res. 14 (2): 4584-4592 (2015)

Received July 14, 2014

Accepted March 5, 2015

Published May 4, 2015

DOI http://dx.doi.org/10.4238/2015.May.4.17

\begin{abstract}
The aim of this study was to explore the existence of a relationship between the rs189037 single nucleotide polymorphism (SNP) of the ataxia telangiectasia mutated (ATM) gene and cognitive impairment in the elderly (aged 60 years and above). In a cohort, 505 residents of Suinung City were consecutively recruited and their cognitive function was measured using a 30-point Mini-Mental State Examination (MMSE). The subjects were divided into cognitive impairment group and control group on the basis of MMSE scores. Presence of the rs189037 SNP variant was examined using polymerase chain reaction-restriction fragment length polymorphism. The prevalence rates of cognitive impairment were $32.7 \%$ in the whole
\end{abstract}


sample. The genotype frequencies of the rs 189037 polymorphism were $33.5 \%(\mathrm{CC}), 50.7 \%(\mathrm{CT})$, and $15.8 \%$ (TT); the $\mathrm{C}$ and $\mathrm{T}$ allele frequencies were 58.8 and $41.2 \%$, respectively. No significant differences in the frequency distributions of the $\mathrm{CC}, \mathrm{CT}$ and TT genotypes were observed between cognitively impaired and control groups. We found that the rs189037 SNP was not directly correlated with cognitive impairment among the elderly Chinese Han population.

Key words: Ataxia telangiectasia mutated gene; rs189037; Cognitive impairment; Single nucleotide polymorphism

\section{INTRODUCTION}

The number of older adults with cognitive impairment is expected to rise dramatically due to the aging population (Brookmeyer and Gray, 2000). Cognitive impairment causes a significant financial burden to society; the total worldwide societal cost of dementia was estimated to be US\$315 billion for 29.3 million demented individuals in 2005, and the cost had increased to US $\$ 422$ billion in 2009, based on a dementia population of 34.4 million persons, including US\$142 billion for informal care (34\%) (Wimo et al., 2010). Furthermore, dementia, a severe cognitive impairment, affects approximately $7 \%$ of the general population older than 65 years, and $30 \%$ of people older than 80 years (O'Brien et al., 2003). Accumulated evidence from epidemiological research strongly supports roles for lifestyle and cardiovascular risk factors in the pathogenesis and development of cognitive impairment (Qiu et al., 2010). These factors include increasing age, low education, smoking, living alone, poor living conditions (Cervilla et al., 2000; Frisoni et al., 2000; Verghese et al., 2003; Tervo et al., 2004), and vascular-related diseases such as hyperlipidemia, hypertension, heart disease, diabetes mellitus, and cerebrovascular disease or stroke (Elias et al., 1997; Kilander et al., 1998; Kivipelto et al., 2001; Ballard et al., 2003).

To date, four genes associated with Alzheimer's disease have been identified: amyloid precursor protein (APP) (Goate et al., 1991), apoE (Strittmatter et al., 1993), PS1 (Sherrington et al., 1995), and PS2 (Levy-Lahad et al., 1995); these are located on chromosomes 21, 19,14, and 1, respectively. Ataxia telangiectasia mutated (ATM) is the gene mutated in the genetic disorder ataxia telangiectasia, symptoms of which include progressive neurological degeneration, immunodeficiency, high cancer incidence, extreme sensitivity to ionizing radiation, ischemic heart disease, and premature aging ( $\mathrm{Li}$ and Swift, 2000). The ATM gene plays roles in antioxidant elevation, telomere protection, and regulation of insulin-like growth factor receptor 1 expression, and its mutation might result in elevated plasma cholesterol and triglyceride levels, insulin resistance, and impaired glucose tolerance in patients with ataxia telangiectasia (Badalian and Kalininal, 1976; Ristow, 2004). In addition, the antioxidant capability is weakened and the level of reactive oxygen species and oxidative stress are increased in knockout mice lacking the $A T M$ gene (Wu et al., 2005). These parameters are known as major contributors to the pathogenesis of senescence and to the development of atherosclerosis risk factors. In turn, atherosclerosis risk factors such as smoking, hypercholesterolemia, diabetes mellitus, and hypertension are also associated with cognitive impairment (Herbig et al., 2006; Mercer et al., 2010). 
It is well known that promoters play a major role in the control of gene expression, and the $A T M$ promoter region is the primary regulator of $A T M$ gene transcription and expression. In our previous studies, we identified a single nucleotide polymorphism (SNP), rs189037, that is located in the promoter region of $A T M$ and is associated with human longevity and coronary stenosis (Chen et al., 2010; Li et al., 2011). Therefore, based on the evidence mentioned above, we carried out a preliminary experiment to examine whether there was an association between the SNP rs189037 in the ATM gene promoter and cognitive impairment.

\section{MATERIAL AND METHODS}

\section{Setting and subjects}

We recruited 554 participants consecutively from eight villages in the Yongxing county of Suining City, Sichuan, China, between October and December 2011. The inclusion criteria were: 1) 60 years of age and above; 2) prior residence in the trial areas for at least 6 months; and 3) available to and capable of giving informed consent. We excluded those subjects who had mental diseases, trauma, or mental retardation, did not complete the 30-item Mini-Mental State Examination (MMSE), or had no blood specimen for genotype detection and laboratory testing. The trial protocol was approved by the Institutional Review Boards at the West China Hospital and was conducted according to the Helsinki Declaration II. Full informed consent (by signature or thumbprint) was obtained from all trial participants. Trained personnel visited all study participants at local neighborhood committees for data collection, e.g., anthropometric measurements and the collection of a blood specimen. Sociodemographic characteristics and lifestyle habits were collected by using a general questionnaire.

\section{Assessment of cognitive impairment}

The MMSE is a major method to screen cognitive function, and covers components of orientation, attention, calculation, language, and recall (Crum et al., 1993). A total maximal score on the MMSE is 30 points and a score of less than 18 points is generally considered to indicate cognitive impairment (Katzman et al., 1988). To minimize error and assure reliability, we 1) reviewed the MMSE scoring systems outlined in a short booklet and a video; 2) observed a geriatrician performing the MMSE assessment (on residents not part of our study); and 3) obtained supervision when performing the MMSE. The MMSE test was thereafter applied to the consented study subjects. In our study, the subjects were divided into cognitive impairment group ( $\leq 17$ points) and control group ( $>17$ points) on the basis of MMSE scores.

\section{Genotype detection}

Genomic DNA was isolated from whole blood drawn from the antecubital vein, using Blood Genomic extraction kits (DP319, TianGen, Beijing, China) according to standard procedures. Genotyping of the SNP rs 189037 was carried out by a polymerase chain reaction (PCR)-restriction fragment length polymorphism detection method. The forward and 
reverse primers were 5'-GCTGCTTGGCGTTGCTT-3' and 5'-CATGCGATTGGCGGTCTG G-3', respectively (Chen et al., 2010). The PCR cycling conditions used were as follows: an initial denaturation step at $94^{\circ} \mathrm{C}$ for 3 min, followed by 30 cycles of denaturation at $94^{\circ} \mathrm{C}$ for $30 \mathrm{~s}$, annealing for $30 \mathrm{~s}$ at $55^{\circ} \mathrm{C}$, and elongation for $30 \mathrm{~s}$ at $72^{\circ} \mathrm{C}$, with a final extension of 5 min at $72^{\circ} \mathrm{C}$. The amplified PCR products were digested with SacII (TaKaRa, Dalian, China) at $37^{\circ} \mathrm{C}$ overnight and resolved on a $10 \%$ polyacrylamide gel and stained with silver nitrate.

\section{Assessment of covariates}

The baseline examination included information on gender, age (years), MMSE score, educational level (illiteracy, primary school, secondary school and above), fasting plasma glucose (FPG, $\mathrm{mM}$ ), systolic blood pressure ( $\mathrm{SBP}, \mathrm{mmHg}$ ), diastolic blood pressure $(\mathrm{DBP}, \mathrm{mmHg})$, triglycerides $(\mathrm{mM})$, total cholesterol $(\mathrm{mM})$, low-density lipoprotein cholesterol (mM), high-density lipoprotein cholesterol (mM), serum uric acid (UA, mM), body mass index (BMI, $\mathrm{kg} / \mathrm{m}^{2}$ ), alcoholism, and histories of smoking, stroke, diabetes mellitus, hypertension, and coronary heart disease. Right arm blood pressure was measured in the sitting or recumbent positions twice to the nearest $2 \mathrm{mmHg}$ using a standard mercury sphygmomanometer by trained physicians. SBP and DBP were calculated by the mean value of the two measurements. BMI was calculated as body weight (in $\mathrm{kg}$ ) divided by the square of height (in meters). FPG, lipid/lipoprotein levels, and UA were determined by technicians in the laboratory of Suining City People's Hospital. The other covariates were collected by using a general questionnaire.

\section{Statistical analysis}

All of the statistical analyses for this study were performed with the SPSS for Windows software package, version 19.5 (SPSS Inc., Chicago, IL, USA). the Pearson chi-square or the Fisher exact test (where an expected cell count was $<5$ ) was conducted to assess whether the genotypic frequencies conformed to Hardy-Weinberg equilibrium, and to compare the genotypic and allelic frequencies between cognitive impairment and control groups (MMSE cut-off point $=18$ ). Multiple-logistic regression models were used to adjust for factors associated with cognitive function. A P value $<0.05$ was considered to be the criterion of statistical significance and all of the $\mathrm{P}$ values were two-sided.

\section{RESULTS}

\section{Baseline characteristics of cognitive impairment}

Of 554 participants, 49 were excluded for no blood specimen for genotyping and laboratory testing (41), incomplete MMSE test (5), or for mental diseases or retardation (3). As a result, a total of 505 participants were included in the present study ( 225 men and 280 women). The mean age was $70.80 \pm 6.74$ years (range, $60-90$ years). The total prevalence rate of cognitive impairment (MMSE scores $<18$ ) was $32.7 \%$ in the whole sample. The prevalence rate among men was $15.6 \%$, while it was $46.4 \%$ among women; the difference was statistically significant $(\mathrm{P}<0.001)$. The basic characteristics of the main demographic and clinical characteristics with cognitive impairment are presented in Table 1. 
Table 1. Baseline characteristics according to cognitive impairment $(\mathrm{N}=505$, means \pm standard deviation).

\begin{tabular}{|c|c|c|c|c|}
\hline \multirow[t]{2}{*}{ Characteristics } & \multicolumn{2}{|c|}{ Cognitive } & \multirow[t]{2}{*}{ Wilcoxon or $\chi^{2}$} & \multirow[t]{2}{*}{$P$ value } \\
\hline & Impairment $(\mathrm{N}=165)$ & Normal $(\mathrm{N}=340)$ & & \\
\hline Age (years) & $73.84 \pm 7.12$ & $69.32 \pm 6.03$ & 75366.0 & 0.000 \\
\hline Gender (male/female, $\mathrm{N}$ ) & $35 / 130$ & $190 / 150$ & 54.05 & 0.000 \\
\hline Score on MMSE & $13.04 \pm 3.32$ & $23.97 \pm 3.60$ & 13695.0 & 0.000 \\
\hline BMI $\left(\mathrm{kg} / \mathrm{m}^{2}\right)$ & $22.32 \pm 3.26$ & $22.48 \pm 3.18$ & 39473.5 & 0.464 \\
\hline $\mathrm{SBP}(\mathrm{mmHg})$ & $153.28 \pm 25.13$ & $150.74 \pm 23.46$ & 84135.0 & 0.340 \\
\hline DBP (mmHg) & $91.94 \pm 15.45$ & $91.95 \pm 15.21$ & 85143.5 & 0.767 \\
\hline $\mathrm{FPG}(\mathrm{mM})$ & $6.21 \pm 2.85$ & $5.81 \pm 2.08$ & 83406.0 & 0.089 \\
\hline $\mathrm{TG}(\mathrm{mM})$ & $1.50 \pm 1.22$ & $1.37 \pm 0.82$ & 85263.5 & 0.623 \\
\hline $\mathrm{TC}(\mathrm{mM})$ & $5.00 \pm 0.98$ & $5.00 \pm 0.90$ & 41229.0 & 0.737 \\
\hline HDL-C (mM) & $1.83 \pm 0.32$ & $1.84 \pm 0.33$ & 85556.5 & 0.763 \\
\hline LDL-C (mM) & $2.50 \pm 0.74$ & $2.52 \pm 0.73$ & 40806.5 & 0.542 \\
\hline $\mathrm{UA}(\mathrm{mM})$ & $282.68 \pm 71.89$ & $285.36 \pm 68.39$ & 41063.5 & 0.658 \\
\hline \multicolumn{5}{|l|}{ Educational levels $(\mathrm{N})$} \\
\hline Illiteracy & $142 / 165$ & $148 / 340$ & & \\
\hline Primary school & $21 / 165$ & $157 / 340$ & 82.76 & 0.000 \\
\hline Secondary school and above & $2 / 165$ & $35 / 340$ & & \\
\hline Alcoholic habits (\%) & $45(27.3)$ & $130(38.2)$ & 5.90 & 0.015 \\
\hline Smoking habits (\%) & $15(9.1)$ & $106(31.2)$ & 29.74 & 0.000 \\
\hline DM (\%) & $12(7.3)$ & $10(2.9)$ & 5.00 & 0.025 \\
\hline History of stroke (\%) & $2(1.2)$ & $5(1.5)$ & 0.05 & 0.816 \\
\hline Hypertension (\%) & $52(31.5)$ & $80(23.5)$ & 3.67 & 0.055 \\
\hline CHD (\%) & $10(6.1)$ & $18(5.3)$ & 0.12 & 0.724 \\
\hline \multicolumn{5}{|l|}{ Genotype } \\
\hline $\mathrm{CC}(\%)$ & $49(29.7)$ & $120(35.3)$ & & \\
\hline CT (\%) & $89(53.9)$ & $167(49.1)$ & 1.59 & 0.451 \\
\hline TT (\%) & $27(16.4)$ & $53(15.6)$ & & \\
\hline
\end{tabular}

Baseline characteristics were compared between those with and without prevalent cognitive impairment. Statistical analyses were performed using the Pearson chi-square or the Fisher exact test (where an expected cell count was $<5$ ) for categorical variables and the unpaired Student $t$-test for continuous variables. In the testing, a $\mathrm{P}$ value $<0.05$ was considered to be statistically significant. MMSE $=$ Mini-Mental State Examination; BMI = body mass index; FPG = fasting plasma glucose; HDL-C = high-density lipoprotein cholesterol; LDL-C: = low-density lipoprotein cholesterol; $\mathrm{SBP}=$ systolic blood pressure; $\mathrm{DBP}=$ diastolic blood pressure; $\mathrm{TG}=$ triglycerides; $\mathrm{TC}=$ total cholesterol; $\mathrm{UA}=$ uric acid; $\mathrm{DM}=$ diabetes mellitus; $\mathrm{CHD}=$ coronary heart disease.

\section{SNP rs189037 genotyping}

The PCR amplification products of rs189037 are shown in Figure 1A; the products were digested by SacII, resolved by $10 \%$ polyacrylamide gel electrophoresis, and stained with silver nitrate. Three fragments of 46, 116, and $125 \mathrm{bp}$ were generated when the CC genotype was present, while two fragments of 125 and $162 \mathrm{bp}$ were generated when the TT genotype was present, and four fragments of $46,116,125$, and $162 \mathrm{bp}$ were generated when the heterozygous CT genotype was present (Figure 1B).

\section{Association between the SNP rs189037 and cognitive function}

The genotypic frequencies of the SNP rs189037 in the promoter region of the ATM gene were $33.5 \% \mathrm{CC}, 50.7 \% \mathrm{CT}$, and $15.8 \% \mathrm{TT}$ in the whole sample. $\mathrm{C}$ and T allele frequencies were 58.8 and $41.2 \%$, respectively. We observed that the genotypic and allelic frequencies of the SNP rs 189037 had no significant difference between the cognitively impaired and control groups (Table 2). Demographic and clinical characteristics grouped by SNP rs189037 are presented in Table 3. 

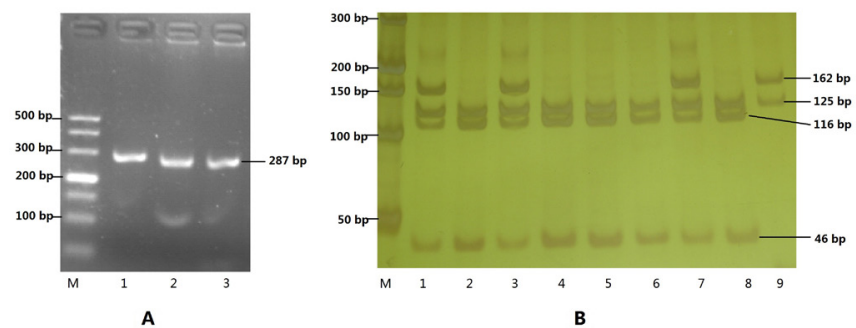

Figure 1. PCR amplification products (A) and polymerase chain reaction-restriction fragment length polymorphism genotyping results (B) of the rs 189037 polymorphism. Lane M: marker; lanes 1, 3, and 7 in (B) represent the CT genotype; lanes $2,4,5,6$, and 8 represent the CC genotype; while lane 9 represents the TT genotype.

Table 2. Association between SNP rs 189037 and cognitive function [case (\%)].

\begin{tabular}{|c|c|c|c|c|c|}
\hline & & \multicolumn{2}{|c|}{ Cognitive } & \multirow[t]{2}{*}{$\chi^{2}$} & \multirow[t]{2}{*}{$P$} \\
\hline & & Impairment $[\mathrm{N}=165(\%)]$ & Normal $[\mathrm{N}=340(\%)]$ & & \\
\hline \multirow[t]{3}{*}{ Genotypes [N (\%)] } & $\mathrm{CC}$ & $49(29.7)$ & $120(35.3)$ & 1.592 & 0.451 \\
\hline & $\mathrm{CT}$ & $89(53.9)$ & $167(49.1)$ & & \\
\hline & TT & $27(16.4)$ & $53(15.6)$ & & \\
\hline \multirow[t]{2}{*}{ Dominant model } & $\mathrm{CC}$ & $49(29.7)$ & $120(35.3)$ & 1.563 & 0.211 \\
\hline & $\mathrm{CT}+\mathrm{TT}$ & $116(70.3)$ & $220(64.7)$ & & \\
\hline \multirow[t]{2}{*}{ Recessive model } & TT & $27(16.4)$ & $53(15.6)$ & 0.050 & 0.823 \\
\hline & $\mathrm{CC}+\mathrm{CT}$ & $138(83.6)$ & $287(84.4)$ & & \\
\hline \multirow[t]{2}{*}{ Alleles } & $\mathrm{C}$ & $187(56.7)$ & $407(59.9)$ & 0.931 & 0.335 \\
\hline & $\mathrm{T}$ & $143(43.3)$ & $273(40.1)$ & & \\
\hline
\end{tabular}

Dominant model: CC vs CT+TT; recessive model: TT vs $\mathrm{CC}+\mathrm{CT}$. $\mathrm{SNP}=$ single nucleotide polymorphism.

Table 3. Baseline characteristics according to genotype ( $\mathrm{N}=505$, means \pm standard deviation).

\begin{tabular}{|c|c|c|c|c|c|}
\hline Characteristics & $\mathrm{CC}$ & $\mathrm{CT}$ & TT & Wilcoxon or $\chi^{2}$ & $P$ value \\
\hline Age (years) & $70.76 \pm 6.82$ & $70.82 \pm 6.78$ & $70.81 \pm 6.55$ & 0.047 & 0.977 \\
\hline Gender (male/female, $\mathrm{N}$ ) & $74 / 95$ & $109 / 147$ & $42 / 38$ & 2.490 & 0.288 \\
\hline Score on MMSE & $20.72 \pm 6.32$ & $20.18 \pm 6.01$ & $20.41 \pm 6.67$ & 1.083 & 0.582 \\
\hline $\operatorname{BMI}\left(\mathrm{kg} / \mathrm{m}^{2}\right)$ & $22.52 \pm 3.14$ & $22.31 \pm 3.23$ & $22.64 \pm 3.27$ & 0.510 & 0.775 \\
\hline $\mathrm{SBP}(\mathrm{mmHg})$ & $154.43 \pm 26.48$ & $149.59 \pm 22.05$ & $151.84 \pm 24.33$ & 2.651 & 0.266 \\
\hline $\mathrm{DBP}(\mathrm{mmHg})$ & $92.86 \pm 16.92$ & $91.00 \pm 13.98$ & $93.01 \pm 15.56$ & 2.185 & 0.335 \\
\hline $\mathrm{FPG}(\mathrm{mM})$ & $5.71 \pm 1.8$ & $6.05 \pm 2.67$ & $6.09 \pm 2.33$ & 0.118 & 0.943 \\
\hline $\mathrm{TG}(\mathrm{mM})$ & $1.38 \pm 0.85$ & $1.49 \pm 1.09$ & $1.25 \pm 0.74$ & 3.582 & 0.167 \\
\hline $\mathrm{TC}(\mathrm{mM})$ & $4.92 \pm 0.86$ & $1.49 \pm 1.09$ & $1.25 \pm 0.74$ & 2.424 & 0.298 \\
\hline HDL-C (mM) & $1.82 \pm 0.29$ & $1.86 \pm 0.35$ & $1.82 \pm 0.30$ & 0.553 & 0.758 \\
\hline LDL-C (mM) & $2.44 \pm 0.68$ & $2.53 \pm 0.78$ & $2.61 \pm 0.68$ & 2.846 & 0.241 \\
\hline $\mathrm{UA}(\mathrm{mM})$ & $278.39 \pm 64.72$ & $285.41 \pm 69.54$ & $294.39 \pm 78.17$ & 2.524 & 0.283 \\
\hline Alcoholic habits (\%) & $57(33.7)$ & $88(34.4)$ & $30(37.5)$ & 0.359 & 0.836 \\
\hline Smoking habits (\%) & $31(18.3)$ & $67(26.2)$ & $23(28.8)$ & 4.621 & 0.099 \\
\hline \multicolumn{6}{|l|}{ Educational levels (N) } \\
\hline Illiteracy & $100 / 169$ & $146 / 256$ & $44 / 80$ & & \\
\hline Primary school & $53 / 169$ & $97 / 256$ & $28 / 80$ & 5.016 & 0.286 \\
\hline $\begin{array}{l}\text { Secondary school } \\
\text { and above }\end{array}$ & $16 / 169$ & $13 / 256$ & $8 / 80$ & & \\
\hline DM $(\%)$ & $5(3.0)$ & $13(5.1)$ & $4(5.0)$ & 1.192 & 0.551 \\
\hline History of stroke (\%) & $1(0.6)$ & $3(1.2)$ & $3(3.8)$ & 4.137 & 0.126 \\
\hline Hypertension (\%) & $53(31.4)$ & $59(23.0)$ & $20(25.0)$ & 3.709 & 0.157 \\
\hline CHD (\%) & $9(5.3)$ & $17(6.6)$ & $2(2.5)$ & 2.019 & 0.364 \\
\hline Cognitive Impairment (\%) & $49(29.0)$ & $89(34.8)$ & $27(33.7)$ & 1.592 & 0.451 \\
\hline
\end{tabular}

Baseline characteristics were compared between different genotypes using the $\chi^{2}$ or the Fisher exact test (where an expected cell count was $<5$ ) for categorical variables and the unpaired Student $t$-test for continuous variables. In the testing, a $\mathrm{P}$ value $<0.05$ was considered to be statistically significant. MMSE = Mini-Mental State Examination; $\mathrm{BMI}=$ body mass index; FPG = fasting plasma glucose; HDL-C = high-density lipoprotein cholesterol; LDL-C = low-density lipoprotein cholesterol; SBP = systolic blood pressure; DBP = diastolic blood pressure; $\mathrm{TG}=$ triglycerides; $\mathrm{TC}=$ total cholesterol; $\mathrm{UA}=$ uric acid; $\mathrm{DM}=$ diabetes mellitus; $\mathrm{CHD}=$ coronary heart disease. 
Multiple-logistic regression analyses were used to adjust for factors associated with cognitive function. The results showed that age, gender, and education levels were factors that impacted cognitive dysfunction $(\mathrm{P}<0.05)$. However, genotypes of the polymorphism rs189037 were not significant factors of cognitive impairment $(\mathrm{P}>0.05$; Table 4$)$.

Table 4. Risk factors for cognitive function in multiple logistic regression analysis.

\begin{tabular}{lccccc}
\hline Characteristics & $\beta$ & $\mathrm{SE}$ & $\mathrm{P}$ & $\mathrm{OR}$ & $95 \% \mathrm{CI}$ \\
\hline Age & 0.119 & 0.019 & 0.000 & 1.127 & $1.085-1.170$ \\
Gender & 1.146 & 0.276 & 0.000 & 3.144 & $1.831-5.399$ \\
Educational level & -1.254 & 0.270 & 0.000 & 0.285 & $0.168-0.484$ \\
Genotype & & & 0.269 & & \\
CT** & 0.324 & 0.254 & 0.201 & 1.383 & $0.841-2.275$ \\
$\quad$ TT* & 0.510 & 0.347 & 0.142 & 1.665 & $0.844-3.287$ \\
Constant & -7.062 & 1.817 & 0.000 & 0.001 & \\
\hline
\end{tabular}

*The $\mathrm{CC}$ genotype was taken as the control group. $\mathrm{SE}=$ standard error; $\mathrm{OR}=$ odds ratio; $\mathrm{CI}=$ confidence interval. Dummy variables were assigned for gender: male $=0$, female $=1$. Dummy variables were assigned for educational levels: illiteracy $=0$, primary school $=1$, secondary school and above $=2$.

\section{DISCUSSION}

Cognitive impairment is a multi-factorial condition, which is influenced both by genetic and demographic factors, and, importantly, by modifiable environmental factors. Recent long-term population-based studies have suggested that vascular risk factors and vascularrelated diseases were risk factors for vascular dementia, and also have an important role in the pathogenesis of cognitive impairment (Tervo et al., 2004). The present study showed that elderly people living in rural areas have a high prevalence of cognitive impairment (32.7\%). This might have been observed because we included mild cognitive impairment participants. The prevalence of cognitive impairment in women (46.4\%) was three times as high as that in men (15.6\%), which is consistent with previous studies (Andersen et al., 1999). There were no significant differences of the frequency distributions of the CC, CT, and TT genotypes of the SNP rs189037 between the cognitively impaired and control groups. Furthermore, multiplelogistic regression analysis showed that genotypes of the SNP rs189037 had no effect on cognitive impairment. Accordingly, the present study indicated that there is no relationship between the SNP rs 189037 in the promoter region of the ATM gene and cognitive impairment.

However, numerous studies have found that the $A T M$ gene was related to risk factors of cognitive dysfunction (Chen et al., 2010; Li et al., 2011). In previous studies, we observed a significant association between the CT genotype of the polymorphism rs189037 and longevity, and individuals with the CT heterozygote genotype had the potential to live longer (Chen et al., 2010). In subsequent experiments, we found that the TT genotype was associated with less severe coronary stenosis, indicating that the TT genotype was a protective factor for coronary heart disease (Li et al., 2011). In addition, ATM heterozygosity in ApoE null mice promotes atherosclerosis and multiple features of metabolic syndrome, including hypertension, hypercholesterolemia, hepatic steatosis, glucose intolerance, and alterations in lipid metabolism (Mercer et al., 2012). Coronary and aortic atherosclerosis and its associated diseases have also been observed in ATM-deficient mice (Herbig et al., 2006), suggesting that a decreased expression level of the $A T M$ gene is a risk factor for vascular diseases.

There are some probable explanations for the negative results of the present study: 1) the development of cognitive dysfunction is the result of both genetic and environmental 
factors, and the effect of the SNP rs189037 might be not robust enough to be detected; 2) all of the participants in this study lived in rural areas and the majority took part in physical activities almost every day, which is a protective factor for cognitive impairment (Laurin et al., 2001), which may modify the clinical expression of cognitive impairment; 3 ) the sample size was limited. The number of TT genotype carriers was only 80 , which might weaken statistical efficacy. We noticed that the CT genotype in the cognitive impaired group was more frequent than in the normal group (53.9 vs 49.1\%), although this did not reach a statistical difference. If the sample size was expanded, a statistically significant difference might be observed. In addition, only the Han population was included in our study; thus, this does not imply that the negative result is applicable to other races. For example, the incidence of a polymorphism in the PSI gene, which is related to Alzheimer's disease, was different in different races (Wragg et al., 1996).

In conclusion, in the present study we did not find an association between the SNP rs 189037 and cognitive impairment in a Chinese Han elderly population from rural areas. Several limitations exist in this study, including the limited sample size and other potential confounders, such as nutrition and socio-economic status. Multi-ethnic and different regional studies with a larger sample size are needed in the future to confirm and expand this result.

\section{Conflicts of interest}

The authors declare no conflict of interest.

\section{ACKNOWLEDGMENTS}

Research supported by the National Natural Science Foundation of China (\#81100148), the Science and Technology Department of Sichuan Province (\#2010SZ0181), the Doctoral Fund of Ministry of Education of China (\#20100181110026), and the Ministry of Health for Public Welfare funds (\#201002011 and \#201302008). We are very grateful to the participants and their legal proxies for participating in the study with patience and cooperation. Meantime, we thank the staff of the Department of Geriatrics Medicine, West China Hospital, Yongxing county of Suining City Government and Suining People's Hospital.

\section{REFERENCES}

Andersen K, Launer LJ, Dewey ME, Letenneur L, et al. (1999). Gender differences in the incidence of AD and vascular dementia. The EURODEM Studies. EURODEM Incidence Research Group. Neurology 53: 1992-1997.

Badalian LO and Kalininal LV (1976). Lipid metabolism disorder in ataxia-telangiectasia. Zh. Nevropatol. Psikhiatr. Im. S. S. Korsikova 76: 655-659.

Ballard C, Rowan E, Stephens S, Kalaria R, et al. (2003). Prospective follow-up study between 3 and 15 months after stroke: improvements and decline in cognitive function among dementia-free stroke survivors $>75$ years of age. Stroke 34: 2440-2444.

Brookmeyer R and Gray S (2000). Methods for projecting the incidence and prevalence of chronic diseases in aging populations: application to Alzheimer's disease. Stat. Med. 19: 1481-1493.

Cervilla JA, Prince M and Mann A (2000). Smoking, drinking, and incident cognitive impairment: a cohort community based study included in the Gospel Oak Project. J. Neurol. Neurosurg. Psychiatry 68: 622-626.

Chen T, Dong BR, Lu Z, Tian B, et al. (2010). A functional single nucleotide polymorphism in promoter of ATM is associated with longevity. Mech. Ageing Dev. 131: 636-640.

Crum RM, Anthony JC, Bassett SS and Folstein MF (1993). Population-based norms for the Mini-Mental State Examination by age and educational level. JAMA 269: 2386-2391.

Elias PK, Elias MF and D'Agostino RB (1997). NIDDM and blood pressure as risk factors for poor cognitive performance: 
the Framingham study. Diabetes Care 20: 1388-1395.

Frisoni GB, Fratiglioni L, Fastbom J, Guo Z, et al. (2000). Mild cognitive impairment in the population and physical health: data on 1435 individuals aged 75 to 95. J. Gerontol. A Biol. Sci. Med. Sci. 55: M322-328.

Goate A, Chartier-Harlin MC, Mullan M, Brown J, et al. (1991). Segregation of a missense mutation in the amyloid precursor protein gene with familial Alzheimer's disease. Nature 349: 704-706.

Herbig U, Ferreira M, Condel L, Carey D, et al. (2006). Cellular senescence in aging primates. Science 311: 1257.

Katzman R, Zhang MY, Ouang-Ya-Qu, Wang ZY, et al. (1988). A Chinese version of the Mini-Mental State Examination; impact of illiteracy in a Shanghai dementia survey. J. Clin. Epidemiol. 41: 971-978.

Kilander L, Andrén B, Nyman H, Lind L, et al. (1998). Atrial fibrillation is an independent determinant of low cognitive function: a cross-sectional study in elderly men. Stroke 29: 1816-1820.

Kivipelto M, Helkala EL, Hänninen T, Laakso MP, et al. (2001). Midlife vascular risk factors and late-life mild cognitive impairment. A population-based study. Neurology 56: 1683-1689.

Laurin D, Verreault R, Lindsay J, MacPherson K, et al. (2001). Physical activity and risk of cognitive impairment and dementia in elderly persons. Arch. Neurol. 58: 498-504.

Levy-Lahad E, Wasco W, Poorkaj P, Romano DM, et al. (1995). Candidate gene for the chromosome 1 familial Alzheimer's disease locus. Science 269: 973-977.

Li A and Swift M (2000). Mutations at the Ataxia-Telangiectasia locus and clinical phenotypes of A-T patients. Am. J. Med. Genet. 92: 170-177.

Li S, Zhang L, Chen T, Tian B, et al. (2011). Functional polymorphism rs189037 in the promoter region of ATM gene is associated with angiographically characterized coronary stenosis. Atherosclerosis 219: 694-697.

Mercer JR, Cheng KK, Figg N, Gorenne I, et al. (2010). DNA damage links mitochondrial dysfunction to atherosclerosis and the metabolic syndrome. Circ. Res. 107: 1021-1031.

Mercer JR, Yu E, Figg N, Cheng KK, et al. (2012). The mitochondria-targeted antioxidant MitoQ decreases features of the metabolic syndrome in ATM+/-/ApoE-/- mice. Free Radic. Biol. Med. 52: 841-849.

O’Brien JT, Erkinjuntti T, Reisberg B, Roman G, et al. (2003). Vascular cognitive impairment. Lancet Neurol. 2: 89-98.

Qiu C, Xu W and Fratiglioni L (2010). Vascular and psychosocial factors in Alzheimer's disease: epidemiological evidence towards intervention. J. Alzheimers Dis. 20: 689-697.

Ristow M (2004). Neurodegenerative disorders associated with diabetes mellitus. J. Mol. Med. 82: 510-529.

Sherrington R, Rogaev EI, Liang Y, Rogaeva EA, et al. (1995). Cloning of a gene bearing missense mutation in early-onset familial Alzheimer's disease. Nature 375: 754-760.

Strittmatter WJ, Saunders AM, Schmechel D, Pericak-Vance M, et al. (1993). Apolipoprotein E: high affinity binding to $\beta$-amyloid and increased frequency of type 4 allele in familial Alzheimer's disease. Proc. Natl. Acad. Sci. U.S.A. 90: 1977-1981.

Tervo S, Kivipelto M, Hänninen T, Vanhanen M, et al. (2004). Incidence and risk factors for mild cognitive impairment: a population-based three-year follow-up study of cognitively healthy elderly subjects. Dement. Geriatr. Cogn. Disord. 17: 196-203.

Verghese J, Lipton RB, Katz MJ, Hall CB, et al. (2003). Leisure activities and the risk of dementia in the elderly. N. Engl. J. Med. 348: 2508-2516 .

Wimo A, Winblad B and Jönsson L (2010). The worldwide societal costs of dementia: Estimates for 2009. Alzheimers Dement 6: 98-103.

Wragg M, Hutton M and Talbot C (1996). Genetic association between intronic polymorphism in presinilin-gene and lateonset Alzheimer's disease. Alzheimer's Disease Collaborative Group. Lancet 347: 509-512.

Wu D, Yang H, Xiang W, Zhou L, et al. (2005). Heterozygous mutation of ataxia-telangiectasia mutated gene aggravates hypercholesterolemia in apoE-deficient mice. J. Lipid Res. 46: 1380-1387. 